\title{
YAP scintillators for resonant detection of epithermal neutrons at pulsed neutron sources
}

\author{
M. Tardocchi ${ }^{\mathrm{a})}$ and G. Gorini \\ INFM, UdR Milano-Bicocca, Milano, Italy and Università degli Studi di Milano-Bicocca, \\ Dipartimento di Fisica “G. Occhialini,” Milano, Italy
}

A. Pietropaolo, C. Andreani, and R. Senesi

INFM, UdR Tor Vergata, Roma, Italy and Università degli Studi di Roma "Tor Vergata," Dipartimento di Fisica, Roma, Italy

N. Rhodes and E. M. Schooneveld

Rutherford Appleton Laboratory, ISIS Facility, Didcot, United Kingdom

(Received 4 May 2004; accepted 24 July 2004; published 1 November 2004)

\begin{abstract}
Recent studies indicate the resonance detector $(\mathrm{RD})$ technique as an interesting approach for neutron spectroscopy in the electron volt energy region. This work summarizes the results of a series of experiments where $\mathrm{RD}$ consisting of $\mathrm{YAlO}_{3}$ (YAP) scintillators were used to detect scattered neutrons with energy in the range $1-200 \mathrm{eV}$. The response of YAP scintillators to radiative capture $\gamma$ emission from a ${ }^{238} \mathrm{U}$ analyzer foil was characterized in a series of experiments performed on the VESUVIO spectrometer at the ISIS pulsed neutron source. In these experiments a biparametric data acquisition allowed the simultaneous measurements of both neutron time-of-flight and $\gamma$ pulse height (energy) spectra. The analysis of the $\gamma$ pulse height and neutron time of flight spectra permitted to identify and distinguish the signal and background components. These measurements showed that a significant improvement in the signal-to-background ratio can be achieved by setting a lower level discrimination on the pulse height at about $600 \mathrm{keV}$ equivalent photon energy. Present results strongly indicate YAP scintillators as the ideal candidate for neutron scattering studies with epithermal neutrons at both very low $\left(<5^{\circ}\right)$ and intermediate scattering angles. () 2004 American Institute of Physics. [DOI: 10.1063/1.1795091]
\end{abstract}

\section{INTRODUCTION}

The intense fluxes of epithermal neutrons available at spallation sources are ideal to investigate both single particle short-time dynamics (high wave vector, $q$, and energy, $\omega$, transfers) and high energy excitations (low $q$ and high $\omega$ transfers) in condensed matter systems. The former studies are nowadays routinely performed with the experimental technique known as deep inelastic neutron scattering (DINS). DINS allows one to measure the single particle momentum distribution and mean kinetic energy in molecular systems and quantum fluids and solids. ${ }^{1,2}$ The DINS kinematical regime is experimentally achieved employing both high energy $(\omega>1 \mathrm{eV})$ and wave vector $\left(q>20 \AA^{-1}\right)$ transfers where the scattering is well described within the impulse approximation (IA). ${ }^{1}$ In the IA picture the scattering process is interpreted in terms of incoherent scattering off individual (self) scatterers of the sample, occurring within a time scale much shorter than the characteristic dynamical time response of the sample. On the other hand, high energy inelastic neutron scattering (HINS) performed at low wave vector $\left(q<10 \AA^{-1}\right)$ and high energy $(\omega>500 \mathrm{meV})$ transfers provides a way to experimentally investigate dispersion relations of high energy excitations in metals, semiconductors

\footnotetext{
${ }^{\text {a) }}$ Author to whom correspondence should be addressed; electronic mail: marco.tardocchi@mib.infn.it
}

and insulators, high-lying molecular rotovibrational states, molecular electronic excitations, and electronic levels in solids. ${ }^{3}$

An important prerequisite for the HINS technique is the need to detect neutrons with final energy in the range $E_{n}$ $=1-100 \mathrm{eV}$ (Ref. 4) in inverse geometry time-of-flight instruments such as VESUVIO operating at the ISIS pulsed neutron source. The standard detection techniques in use for DINS experiments employing either ${ }^{3} \mathrm{He}$ gas detectors or ${ }^{6} \mathrm{Li}$ glass scintillators as neutron counters become unpractical above, say $E_{n}=10 \mathrm{eV}$ due to the $1 / v$ dependence of the neutron absorption cross sections $(v$ being the neutron velocity). ${ }^{5,6}$ For this reason, in the last years a different approach for the detection of neutrons with electron volt energies has been developed, namely, the resonance detector (RD) technique. ${ }^{7}$

In the RD technique ${ }^{7,8}$ one considers an inverse geometry time-of-flight instrument set up in the configuration where a resonant foil, on the second flight path, selects the energy of the scattered neutrons via resonance absorption in specific isotopes, such as ${ }^{238} \mathrm{U}$ and ${ }^{197} \mathrm{Au}$. The foil strongly absorbs neutrons within a narrow energy interval around nuclear resonances and a photon detector tags the arrival time of the absorbed neutron by detecting the prompt radiative capture $\gamma$-ray cascade. The kinematics of the scattering event is eventually reconstructed through the time-of-flight technique. Early measurements with different types of RD 
setup have been performed to cover both the low $(<50 \mathrm{keV})$ (Ref. 9) and high (>800 keV) (Ref. 5) photon energy range. These have indicated that the increase of background with final neutron energies is the main limitation to the detection of epithermal neutrons of energies above few electron volts. Previously to this work the best results were obtained with cadmium-zinc-telluride semiconductor detectors which allow room-temperature operation with good photon detection efficiency in the range $20-200 \mathrm{keV}$. These results indicated that even without any energy selection the signal-tobackground ratio exceeded the conventional ${ }^{6} \mathrm{Li}$-glass detectors. ${ }^{6}$ In this case selection of the photon energy further improved the signal-to-background ratio of the measurement but with a loss of signal intensity. ${ }^{10}$ The performance of the VESUVIO instrument operating in the RD configuration is further discussed in the present paper with the use of a different photon detector, namely, a $\mathrm{YAlO}_{3}$ (YAP) scintillator, hereafter named YAP.

YAP scintillators are normally employed for photon spectroscopy in different fields including Mössbauer spectroscopy, ${ }^{11} \mathrm{x}$-ray imaging, ${ }^{12}$ and medical applications. ${ }^{13}$ In this work, this device combined with a natural uranium analyzer foil has been employed for epithermal neutron detection. This article describes the response of the YAP detector to the radiative capture $\gamma$ emission from a natural uranium analyzer foil. The YAP performance has been characterized during DINS measurements from a reference polycrystalline $\mathrm{Pb}$ sample. Data where acquired with a biparametric data acquisition which allowed simultaneous measurements of neutron time-of-flight and $\gamma$ pulse height (energy) spectra. ${ }^{10}$ The analysis of the $\gamma$ pulse height and neutron time-of-flight spectra permitted to identify the signal and background components. The improvement of the signal-to-background ratio that can be achieved by raising the pulse height threshold is quantified and discussed in Secs. IV and V. Two applications of the RD are finally presented in Sec. VI.

\section{EXPERIMENT}

The experiment has been performed on the VESUVIO inverse geometry neutron spectrometer ${ }^{14}$ which operates at the ISIS spallation neutron source. On the VESUVIO beamline a water moderator at ambient temperature provides a white incident neutron beam. The incident neutron spectrum peaks at about $0.03 \mathrm{eV}$ and has an inverse energy dependence $\left(1 / E_{n}\right)$ in the epithermal region. VESUVIO usually operates with the filter difference technique ${ }^{15}$ where the detection of the scattered neutrons is performed by ${ }^{6} \mathrm{Li}$ glass scintillators located in both back- and forward-scattering positions. ${ }^{14}$ In the present experiment the instrument was, instead, set up to operate in the resonance detector (RD) configuration. In this case, scattered neutrons were recorded by a suitable detection system consisting of a combination of an analyzer foil and a photon detector.

The detection system was located on the secondary flight path at a distance $L_{1}=0.30 \pm 0.01 \mathrm{~m}$ from the sample and a scattering angle $2 \theta=90^{\circ} \pm 5^{\circ}$. A schematics of the experimental setup of the measurement is shown in Fig. 1. The

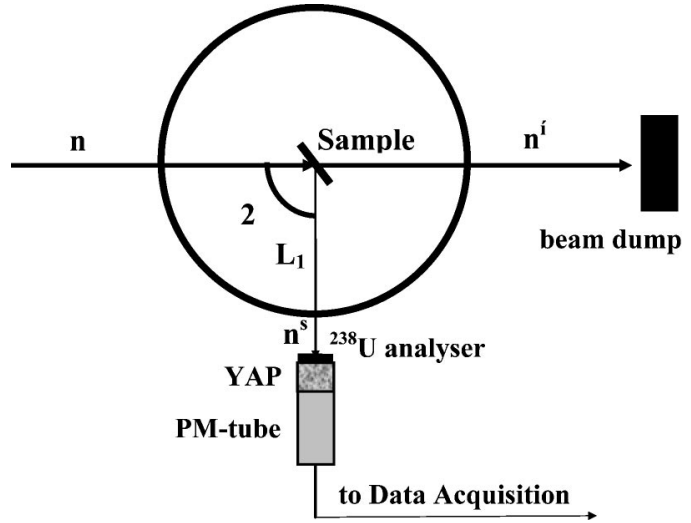

FIG. 1. Schematics top view of the experimental setup of the measurement performed on the Vesuvio spectrometer. The figure shows the incoming $(n)$, outgoing $\left(n^{\prime}\right)$, and scattered $\left(n^{s}\right)$ neutron beam together with the detector system. The detector system is made of an analyzer foil $\left({ }^{238} \mathrm{U}\right)$ viewed from a YAP scintillator coupled to a photomultiplier tube (PM-tube), which sends signals to the Data Acquisition.

incident neutron beam $(n)$ impinges on the sample, placed inside an aluminum vacuum chamber, located at a distance $L_{0}=11.055 \mathrm{~m}$ (primary flight path) from the moderator. The neutron beam is partly transmitted $\left(n^{\prime}\right)$ toward the beam dump, placed five meters beyond the sample position, and partly scattered $\left(n^{s}\right)$. In the present experiment the sample was a $1 \mathrm{~mm}$ thick $\mathrm{Pb}$ metallic slab $\left(2.5 \times 3.5 \mathrm{~cm}^{2}\right)$, supported by an aluminum frame. Scattered neutrons of specific energies are selected via resonance absorption in an analyzer foil. The latter was natural uranium $\left({ }^{238} \mathrm{U}\right)$ in the form of a metallic foil of $2.4 \times 3.5 \mathrm{~cm}^{2}$ area and $60 \mu \mathrm{m}$ thickness. The chosen thickness ensured both good neutron absorption efficiency and low self-absorption of the radiative capture $\gamma$ rays. The foil was attached to a photon detector which recorded the prompt radiative capture $\gamma$ rays emitted by the foil. The photon detector was a yttrium aluminum perovskite $\left(\mathrm{YAlO}_{3}\right)$ scintillator, YAP, doped with cerium. It was directly coupled to a photomultiplier tube and the analog signal sent to the data acquisition system for data recording.

YAP is a fast, mechanically and chemically resistant scintillator material, with mechanical properties enabling precise machining to many different shapes. The inorganic scintillator is nonhygroscopic, glasslike, with a high density $\left(5.55 \mathrm{~g} / \mathrm{cm}^{3}\right)$ but a low effective atomic number $(Z=36)$. Main characteristics of this scintillation material are a good light yield $(\approx 18000$ photons $/ \mathrm{MeV})$ and a short decay time $(\tau=27 \mathrm{~ns})$ at a wavelength of maximum emission of $350 \mathrm{~nm} .{ }^{16}$ The material is relatively stable over a wide temperature range. Its chemical composition is such that no neutron resonances are present in the energy range of interest $(1-200 \mathrm{eV})$. The YAP crystal used in this experiment was manufactured by Bicron (Saint Gobain London, UK) with a cylindrical shape ( $35 \mathrm{~mm}$ diameter by $6.4 \mathrm{~mm}$ thickness). In order to investigate the behavior of the scintillator in a neutron and $\gamma$ enriched environment, the detector was set up to operate without any neutron or $\gamma$ shielding. The crystal was glued to a standard $51 \mathrm{~mm}$ photomultiplier tube made by Electron Tubes Limited (Middlesex, UK) (type 9954) with a bialkali photocathode. The thickness of the crystal was 


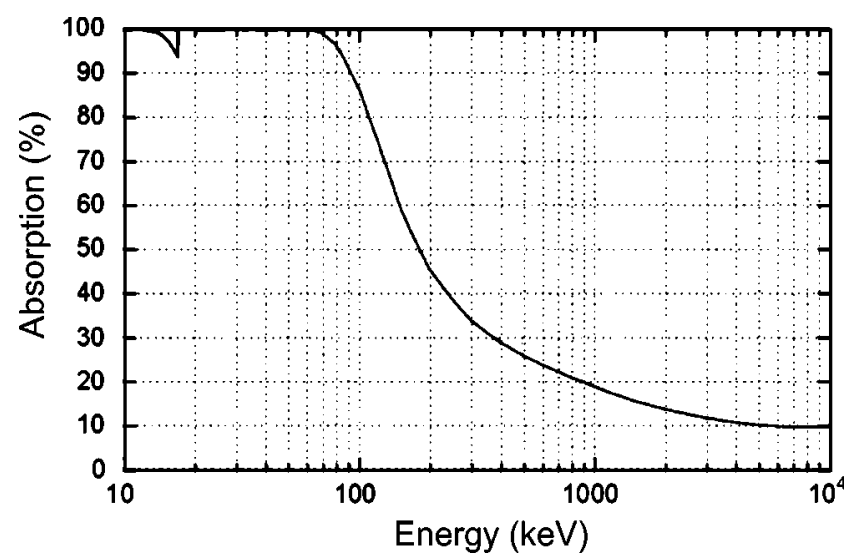

FIG. 2. Photon interaction probability for a $0.6 \mathrm{~mm}$ thick YAP crystal (Ref. 17).

somewhat arbitrary but represents a compromise between the need for good detection efficiency to the $\gamma$ emission following neutron capture in the analyzer foil and low neutron scattering in the crystal. In Fig. 2 the total absorption probability curve for a $6.4 \mathrm{~mm}$ thick YAP crystal is plotted in the $10-1000 \mathrm{keV}$ photon energy range. Below $100 \mathrm{keV}$ photoelectric interaction is the dominant process for energy deposition in the crystal and guarantees $100 \%$ total absorption probability. The edge in the absorption efficiency located at $17 \mathrm{keV}$ corresponds to the $K_{\alpha}$ edge of yttrium. Above about $200 \mathrm{keV}$ compton interaction becomes the dominant mechanism for energy deposition in the crystal, providing a significant absorption probability (about $10 \%$ above $1 \mathrm{MeV}$ ).

In the RD technique the detection of scattered neutrons via a photon detector consists of two separate processes: (i) resonant neutron capture in the analyzer foil and (ii) detection of the prompt radiative capture $\gamma$ rays. The first process selects the mean energy of the absorbed neutron and sets the neutron absorption efficiency. The second step provides the neutron counting and affects the signal-to-background ratio of the measurements. The intensity of the resonance cross section defines, for a given thickness of the analyzer foil, the neutron absorption efficiency while the resonance width contributes to the overall spectrometer energy resolution. ${ }^{7}$ Thus important requirements for the choice of the best analyzer foil are well separated and intense resonance cross sections ( $\sigma_{R}$, defined as the cross section at the peak) with intrinsic narrow widths $\left[\Gamma_{R}\right.$, expressed as full width at half maximum (FWHM)]. ${ }^{18}$ A systematic investigation ${ }^{18}$ of a large variety of nuclear resonances has indicated that ${ }^{238} \mathrm{U}$ is the nuclide showing most intense $\left(\sigma_{R}>10^{4} \mathrm{~b}\right)$ and narrow $\left(\Gamma_{R}<60 \mathrm{meV}\right)$ neutron resonances in the energy range of interest for epithermal neutron spectroscopy, i.e., $1-200 \mathrm{eV}$ (Table I). The intrinsic resonance width is broadened, at a certain finite temperature, by the lattice thermal vibration of the nuclides in the analyser foil (Doppler broadening).

The $\gamma$-ray emission following neutron absorption in ${ }^{238} \mathrm{U}$ consists of many lines spanning a wide spectrum, ranging from $11 \mathrm{keV}$ up to $4.060 \mathrm{MeV}$ which is the neutron binding energy in ${ }^{238} \mathrm{U}$. Earlier measurements with cadmium zinc telluride (CZT) detectors ${ }^{6,10}$ have shown that there is also a significant prompt $\mathrm{x}$-ray emission at the uranium character-
TABLE I. Mean energy $\left(E_{R}\right)$, intensity $\left(\sigma_{R}\right)$ and intrinsic width $\left(\Gamma_{R}\right)$ of ${ }^{238} \mathrm{U}$ neutron resonances in the $1-200 \mathrm{eV}$ neutron energy range (Ref. 19).

\begin{tabular}{ccc}
\hline \hline$E_{R}(\mathrm{eV})$ & $\sigma_{R}(\mathrm{~b})$ & $\Gamma_{R}(\mathrm{eV})$ \\
\hline 6.671 & 23564 & 0.025 \\
20.87 & 37966 & 0.034 \\
36.68 & 42228 & 0.057 \\
66.02 & 20134 & 0.048 \\
80.73 & 2297 & 0.027 \\
102.5 & 18989 & 0.094 \\
116.9 & 11845 & 0.048 \\
189.7 & 12228 & 0.197 \\
\hline \hline
\end{tabular}

istic lines $(95,98$, and $112 \mathrm{keV})$. The expected energies and relative intensities of the radiative capture $\gamma$ rays following thermal neutron absorption in ${ }^{238} \mathrm{U}$ are shown in Fig. $3 .{ }^{20}$ From this figure one can observe that most of the photon emission (about 80\%) occurs at photon energies below $1 \mathrm{MeV}$.

As in a previous work with CZT photon detectors ${ }^{10}$ a dedicated data acquisition system was specifically set up in order to acquire simultaneous measurements of both the neutron time-of-flight (TOF) and the $\gamma$ pulse height (energy) spectra. This set up was achieved by modifying the standard VESUVIO data acquisition electronics (DAE), which normally records TOF spectra through an array of ${ }^{6} \mathrm{Li}$ detectors. The modified DAE was able to record simultaneously both the neutron TOF and photon pulse height values for each photon event (hereafter named biparametric acquisition). The main elements of the signal processing and biparametric acquisition are briefly described below. The negative analog signal from the photomultiplier tube was first integrated with a $270 \mathrm{~ns}$ integration time and thereafter inverted. The positive analog signal was then further amplified by a timing filter amplifier which was set with a gain of 40 and an integration time of $500 \mathrm{~ns}$. The amplified signal was sent to a 13 bit analog-to-digital converter (ADC) of Nuclear Instrumentation Module (NIM) standard produced by FAST ComTec (Oberhaching, Germany). The ADC operated with a fixed conversion time of $500 \mathrm{~ns}$ and an internal lower level discriminator set to the minimum level (about $70 \mathrm{keV}$ during

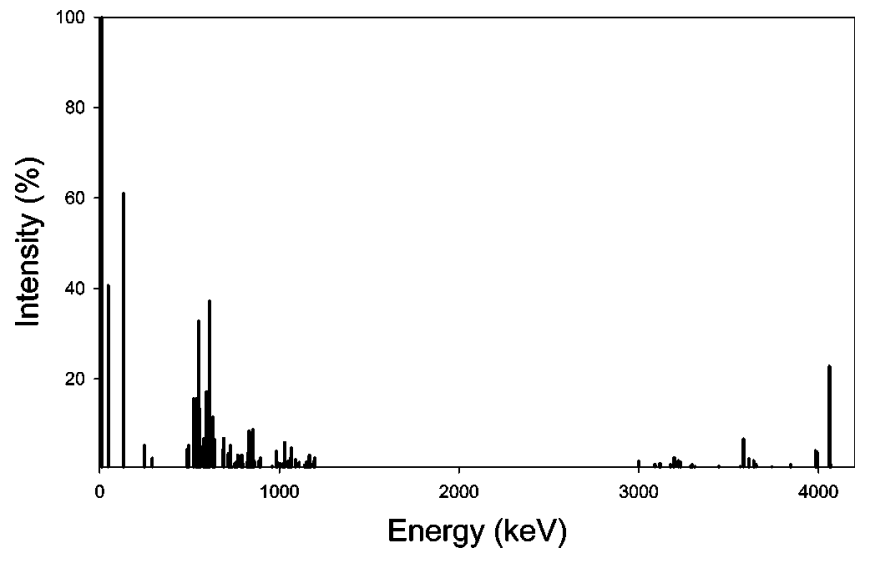

FIG. 3. Relative intensities of the $\gamma$ spectrum following thermal neutron capture in ${ }^{238} \mathrm{U}$. Only the $\gamma$ lines with intensity above $0.5 \%$ are plotted (Ref. 20). 
data acquisition) compatible with acceptable dead time values. The digital output from the ADC was fed into the modified DAE resulting in a biparametric acquisition (TOF and energy). Due to the limited memory in the DAE, the number of channels was chosen to be 1627 for the TOF spectrum and 512 for the pulse height spectrum. A fixed energy bin was chosen for the pulse height spectrum while a variable time bin was used to cover the entire TOF region with adequate time resolution. The chosen time bins values where $0.25 \mu \mathrm{s}$ in the time region 30-260 $\mu \mathrm{s}, 0.5 \mu \mathrm{s}$ in $260-500 \mu \mathrm{s}, 5 \mu \mathrm{s}$ in 500-1500 $\mu \mathrm{s}$ and variable bins in 1500-20000 $\mu \mathrm{s}$. These choices provided adequate energy and time resolution for the measurement.

\section{INSTRUMENT CALIBRATIONS}

The data presented in this article refers to a single run of $11 \mathrm{~h}$, corresponding to an integrated proton beam current of $1936 \mu \mathrm{A}$ h. The collected biparametric data contains dual information. By introducing a selection window on the TOF axis and projecting the data along the $\gamma$ energy axis one obtains the $\gamma$ energy spectrum corresponding to the selected neutron TOF window. In a complementary way, the TOF spectrum corresponding to a selected $\gamma$ energy window can be obtained. Two independent calibrations are therefore needed for each energy and time chain of the biparametric data acquisition system. The two distinct calibrations are described in Secs. III A and III B.

\section{A. Energy calibration}

Absolute calibration of the pulse height values recorded by the biparametric acquisition was performed with a ${ }^{57} \mathrm{Co}$ source. The source provided two $\gamma$ lines of energies 122 and $136 \mathrm{keV}$. The pulse height spectrum was obtained integrating the biparametric spectrum over the entire time axis and projecting the data along the energy axis [Fig. 4(a)]. The main peak, located at about channel (chn.) 60, corresponds to the full-energy deposition peak. This receives the contribution of both the $122 \mathrm{keV}$ (relative intensity, $I \approx 90 \%$ ) and $136 \mathrm{keV}(I \approx 10 \%) \gamma$ lines of the ${ }^{57} \mathrm{Co}$ source. The modest energy resolution of the detector, about $22 \mathrm{keV}$ (FWHM) at $122 \mathrm{keV}$, prevents separating the two lines. The compton shoulder is visible at about chn. 18, above the ADC lower level discriminator set at chn. 12. The structure around chn. 50 can be likely ascribed to characteristic $x$-ray escape peaks of $\mathrm{Ce}\left(K_{\alpha 1}=34.7 \mathrm{keV}, K_{\alpha 2}=34.3 \mathrm{keV}\right)$. Cerium $(Z=58)$, which is present in the YAP scintillator as an activator, shows the highest energy X-ray levels among the $\mathrm{Y}(Z=39), \mathrm{Al}(Z$ $=13)$, and $\mathrm{O}(Z=8)$ atoms of the YAP crystal. The main full energy peak is used as one calibration point for the pulse height axis of the biparametric acquisition. A second calibration energy point is provided by the lead biparametric measurement as described below.

Lead is used as a calibrating sample in DINS measurements because of its well-known scattering function. The $\gamma$ pulse height spectrum, obtained integrating the $\mathrm{Pb}$ sample biparametric data in the TOF region 400-800 $\mu$ s, is shown in Fig. 4(b). The selected TOF region corresponds to incident neutron energies in the range $1-4 \mathrm{eV}$. The broad peak
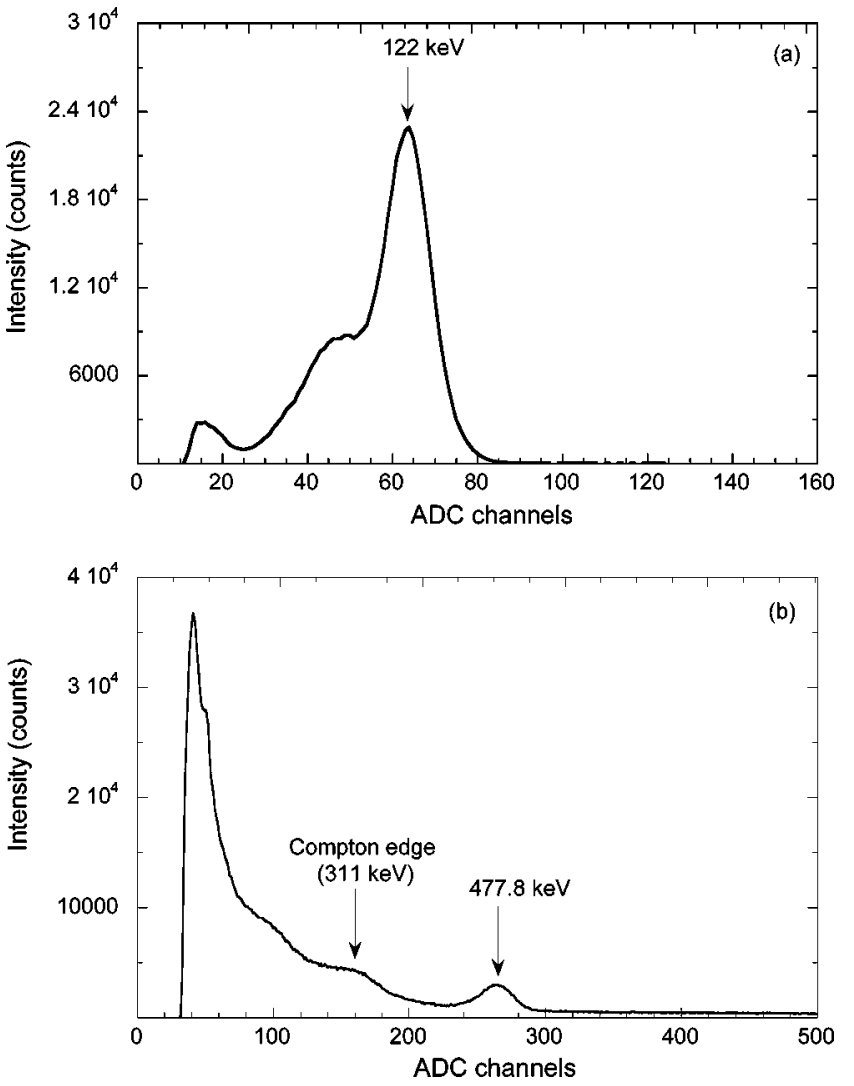

FIG. 4. Pulse height spectra from a ${ }^{57} \mathrm{Co}$ source (top) and $\mathrm{Pb}$ sample measurement (bottom). The pulse height spectrum from the ${ }^{57} \mathrm{Co}$ source is obtained integrating the biparametric data at all times and projecting the data along the energy axis. The pulse height spectrum from the $\mathrm{Pb}$ sample measurement is obtained by projecting the biparametric data along the energy axis in the time interval $400-800 \mu \mathrm{s}$.

around chn. 280 can be ascribed to the full energy absorption of $\gamma$ rays of energy $477.8 \mathrm{keV}$, which are produced via the reaction ${ }^{10} \mathrm{~B}(n, \alpha){ }^{7} \mathrm{Li}$ and the following decay of ${ }^{7} \mathrm{Li}$ nuclide to the ground state. Boron is used in the walls of the experimental hall and in the beam dump to absorb thermal neutrons. We note that, due to the low absorption probability of the YAP detector at such energies the majority of $477.8 \mathrm{keV}$ $\gamma$ rays deposit their energy via compton scattering. The compton edge of the ${ }^{10} \mathrm{~B}$ photopeak is located at $311 \mathrm{keV}$ and shows up with its characteristic shoulder.

The two full energy peaks provided by the pulse height measurements of the ${ }^{57} \mathrm{Co}$ source [Fig. 4(a)], and of the ${ }^{10} \mathrm{~B}$ neutron capture [Fig. 4(b)], respectively, have been used to assign a linear energy scale to the $\gamma$ energy axis of the biparametric data acquisition system. The result of the calibration provides an offset of about $10 \mathrm{keV}$ and a slope of $1.8 \mathrm{keV} / \mathrm{chn}$. The ${ }^{10} \mathrm{~B}$ background peak at $477.8 \mathrm{keV}$ also provides an absolute pulse height reference during the $\mathrm{Pb}$ measurement which has been used to monitor the stability of the gain of the photomultiplier tube and the drifts of the electronics. By analyzing pulse height spectra at different TOF intervals one can check for possible shifts of the $477.8 \mathrm{keV}$ peak. This was done by comparing pulse height spectra obtained integrating TOF values in $50 \mu$ s consecutive intervals. The result indicates that the ${ }^{10} \mathrm{~B}$ peak shifts upward by about $4 \%$ over the whole TOF region. This shift 
can be attributed to an increase of the photomultiplier tube gain. The gain value is higher at the short TOF values when the YAP records the highest count rate, and decreases until it reaches its minimum (steady state) value at about $1000 \mu$ s. The size of the effect, which is a well-known behavior of photomultiplier tubes subject to rapidly varying count rate conditions, ${ }^{21}$ is at an acceptable level for the purpose of this work.

\section{B. Time calibration}

The measured neutron TOF values represent the difference between the multiple stops and the start signals of the neutrons provided by the $\gamma$ detector and spallation source, respectively. Although in the RD technique the stop signal is given by the detection of the radiative capture $\gamma$, the time of the arrival of the $\gamma$ can be assumed to be equal to the time of the absorption of the neutron in the analyzer foil. The timeof-flight value $t_{\text {res }}$ of a neutron scattered from the $\mathrm{Pb}$ sample with final velocity $v_{\text {res }}$ can thus be accurately calculated using the equation

$$
t_{\text {res }}=t_{0}+\frac{L_{0}}{v_{0}}+\frac{L_{1}}{v_{\text {res }}}
$$

here $t_{0}$ is a fixed time delay provided by the ISIS spallation source, $L_{0}$ and $L_{1}$ are the primary and secondary flight paths, respectively, and $v_{0}$ the initial neutron velocity. A value of $L_{0}=11.055 \pm 0.021 \mathrm{~m}$ on VESUVIO was provided by previous calibration measurements. ${ }^{15}$ The final and initial neutron velocities are related by the kinematics of the scattering reaction, which in the case of free recoil can be written $\mathrm{as}^{22}$

$$
\frac{v_{\text {res }}}{v_{0}}=\frac{\cos (2 \vartheta)+\left[(M / m)^{2}-\sin ^{2}(2 \vartheta)\right]^{0.5}}{M / m+1},
$$

where $2 \theta$ is the scattering angle, $M$ the mass of the recoiling $\mathrm{Pb}$ atom in the sample ( $M=207.2$ a.m.u.), and $m$ the neutron mass. From a system with Eqs. (1) and (2) $t_{\text {res }}$ can be expressed as a function of the unknown variables $t_{0}, L_{1}$, and $2 \theta$. A general description of the calibration procedure in the standard configuration can be found in Ref. 15. In this experiment we have determined from direct measurements the values of $L_{1}=30 \pm 2 \mathrm{~cm}$ and $2 \theta=90 \pm 2^{\circ}$, and estimated the best value of $t_{0}$ with the time calibration procedure described below. From the biparametric data the TOF spectrum corresponding to a selected $\gamma$ energy window can be found by projecting the data along the TOF axis. This is shown in Fig. 5 in the time region 50-340 $\mu$ s for a selected $\gamma$ energy window above $600 \mathrm{keV}$. The peaks marked in the spectrum can be ascribed to recoil from $\mathrm{Pb}$ atoms registered via neutron absorption in the ${ }^{238} \mathrm{U}$ analyzer foil at different resonance energies. Seven peaks can be identified corresponding to the ${ }^{238} \mathrm{U}$ resonances going from 6.67 up to $189.7 \mathrm{eV}$. The measured time-of-flight values were corrected for the delay value $t_{0}$, which was determined by fitting the measured positions in time of each resonance $t_{\exp }$ to the calculated values $t_{\text {res }}$ indicated with dashed lines in Fig. 5. The best linear fit yields a value of $t_{0}=-5.9 \pm 0.5 \mu \mathrm{s}$ and gives good agreement between the calculated and measured position for all the observed resonances. A sensitivity test of the calibration procedure to the parameters $L_{1}$ and $2 \theta$ has shown that an uncertainty of

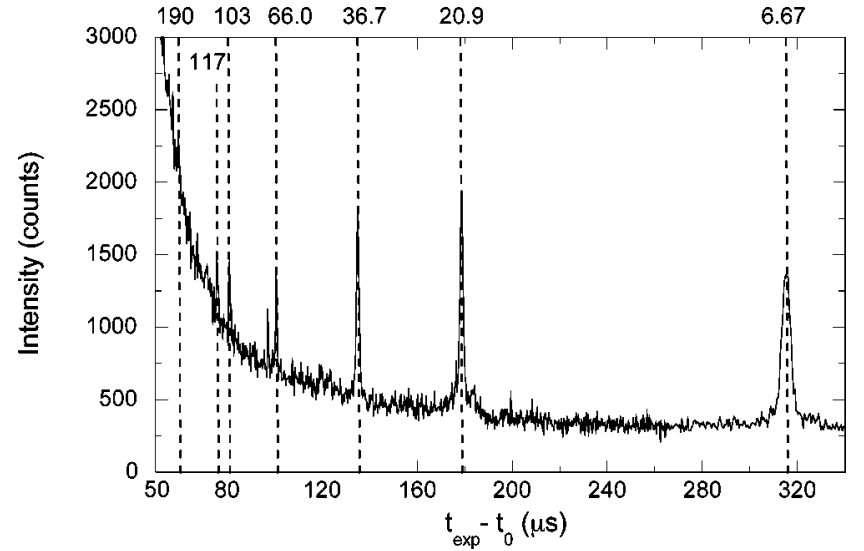

FIG. 5. Time of flight spectrum from the $\mathrm{Pb}$ sample obtained by projecting the biparametric data along the time axis for $\gamma$ energies above $600 \mathrm{keV}$. The expected positions of the ${ }^{238} \mathrm{U}$ resonances are indicated by dashed vertical lines. The label above each line is the corresponding neutron energy in electron volts.

$\pm 5 \mathrm{~cm}$ on $L_{1}$ and an uncertainty of $\pm 20^{\circ}$ on $2 \theta$ give a systematic variation in $t_{0}$ of $\pm 0.6 \mu \mathrm{s}$ and $\pm 0.2 \mu \mathrm{s}$, respectively. This accuracy level is acceptable for the purpose of this study.

\section{DATA ANALYSIS}

The collected biparametric data contains the dual information on the neutron TOF and $\gamma$ pulse height spectra. The data analysis aims to characterize the YAP response by identifying the main signal and background components associated to the absorption of resonant and non-resonant neutrons in the analyzer foil. This is done through the analysis of the $\gamma$ pulse height spectra corresponding to different neutron TOF intervals. Subsequently a lower level discrimination (LLD) in the $\gamma$ energy is introduced in order to produce the corresponding TOF spectrum. The aim here is to investigate the change in the signal-to-background ratio of the TOF measurement as well as in the signal counting statistics.

The TOF spectrum from the $\mathrm{Pb}$ polycrystalline sample generated with a LLD value of $600 \mathrm{keV}$ is shown in a loglog plot in Fig. 6 in the entire TOF region. The plot shows an

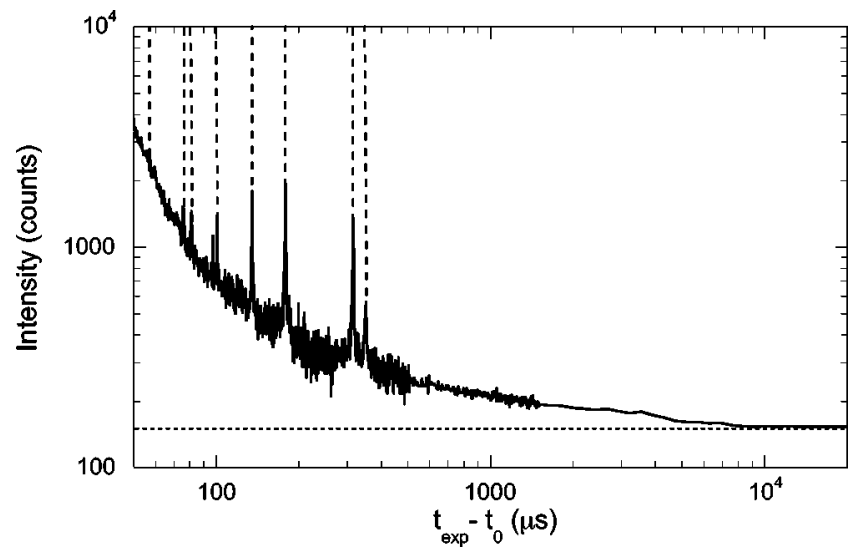

FIG. 6. Same plot as Fig. 5 but plotted in a log scale in the time interval 50-20000 $\mu$ s. The vertical dashed lines indicate the $\mathrm{Pb}$ recoil peaks. The radioactivity level induced by the uranium analyser foil is indicated by the horizontal dashed line. 
overall background with a characteristic power law curve. Two distinct time regions can be identified: (i) $t<800 \mu \mathrm{s}$ where recoil from $\mathrm{Pb}$ atoms is detected via resonant absorption of epithermal neutrons $\left(E_{n}>1 \mathrm{eV}\right)$ by the analyzer foil; (ii) $t>800 \mu \mathrm{s}$ corresponding to the arrival times of lower energy neutrons $\left(E_{n}<1 \mathrm{eV}\right)$. The horizontal dashed line in Fig. 6 indicates the constant background level registered by the YAP, which is due to the analyzer foil radioactivity. The signal in the TOF spectrum is represented by $\mathrm{Pb}$ recoil peaks registered via resonance absorption in the ${ }^{238} U$ analyzer foil at different final neutron energies. Seven peaks corresponding to neutron resonances going from 6.67 up to $189.7 \mathrm{eV}$ can be distinguished from the background (Fig. 5). The resonance at $80.7 \mathrm{eV}$ is not distinguishable in the plot due to the small value of the cross section. The intensity of each $\mathrm{Pb}$ recoil peak in the time channel of width $\Delta t$ centered at the time of flight $t$ is proportional to the quantity

$$
I\left(E_{0}\right) \frac{d E_{0}}{d t} \Delta t\left(\frac{d^{2} \sigma}{d E_{1} d \Omega}\right) \Delta E_{1} \Delta \Omega \eta\left(E_{1}\right),
$$

where $I\left(E_{0}\right) d E_{0} / d t$ is incident neutrons flux (measured in $\left.\mathrm{s}^{-2} \mathrm{~cm}^{-2}\right), d^{2} \sigma / d E_{1} d \Omega$ the $\mathrm{Pb}$ double differential scattering cross section, $\Delta \Omega$ the solid angle viewed from the analyzer foil, $E_{1}$ and $\Delta E_{1}$ the mean energy and width of the resonance cross section, respectively, and $\eta\left(E_{1}\right)$ the overall detection efficiency. For a RD $\eta\left(E_{1}\right)$ is, at a first approximation, given by the product of the probability for a neutron of energy $E_{1}$ to be absorbed in the analyzer foil, $\left(1-e^{-N \cdot \sigma_{R}^{\text {eff }} \cdot d}\right)$, times the detection efficiency of the radiative prompt $\gamma$ emission, $\eta\left(E_{\gamma}\right)$. Here $N$ is the analyzer foil atomic density, $\sigma_{R}^{\text {eff }}$ the effective absorption cross section at the peak, i.e., the $\sigma_{R}$ values of Table I corrected for the Doppler effect at room temperature ${ }^{23}$ and $d$ the thickness of the foil. The line shape of each recoil peak is due to the $\mathrm{Pb}$ compton profile convoluted with the instrument energy resolution. ${ }^{6}$ For a detail description of the $\mathrm{Pb}$ scattering function at room temperature we refer to Ref. 15. The energy resolution is dominated by the analyzer foil whose main contributions are the intrinsic resonance cross section width, the Doppler broadening caused by the thermal lattice motion and the thickness of the foil. The shrinking of the resonance peaks as one moves toward low times-of-flight is mainly caused by the nonlinear kinematic relationship between neutron time-of-flight and neutron energy. This set the requirements on the DAE time resolution in order to perform a line shape analysis of the higher energy resonances. The present lower limit of $250 \mathrm{~ns}$ on the bin width of the DAE will be superseded by the planned DAE which will accept bin width as low as $100 \mathrm{~ns}$.

The background under the recoil peak can be divided into an environmental component, consisting of $\mathrm{x}$-rays and $\gamma$-rays with a time structure related to the neutron beam, and a constant radioactivity component, which has a flat time spectrum as shown in Fig. 6 by the asymptotic behavior of the TOF spectrum at long times. The environmental $\gamma$ background is generated by neutrons absorbed in the various materials present in the experimental hall, e.g., in the beam dump. A significant contribution to the $\gamma$ background is also represented by neutrons which scatter several times in the experimental hall before being absorbed in the ${ }^{238} \mathrm{U}$ analyzer

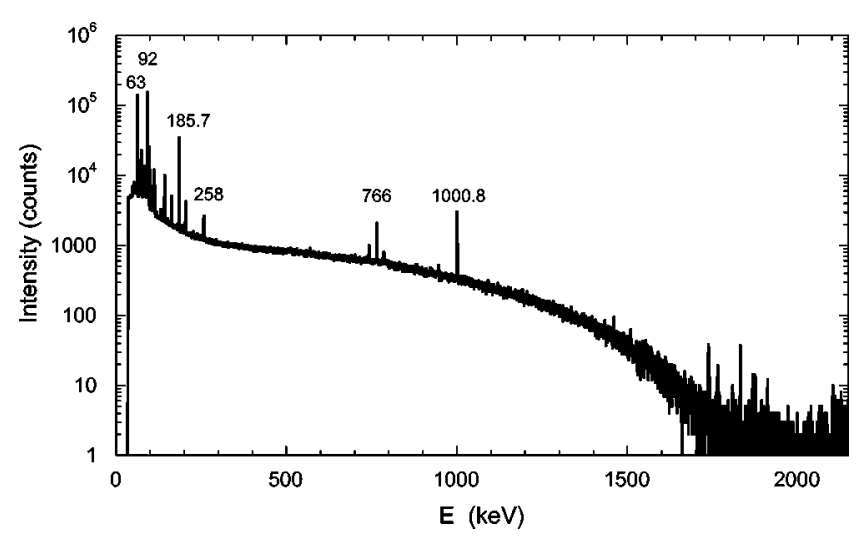

FIG. 7. Pulse height spectrum of the ${ }^{238} U$ analyzer foil recorded with a Germanium detector. The mean energies of some of the most prominent peaks are indicated.

foil. Finally, a fraction of $\gamma$ rays is also known to come directly from the cell and sample itself whenever they contain isotopes with a high absorbing radiative neutron cross section. This sample dependent background in the case of $\mathrm{Pb}$ in an $\mathrm{Al}$ cell is a small fraction of the total background; it can represent a significant fraction for samples containing hydrogen isotopes. ${ }^{24}$ The radioactivity of the analyzer foil was separately measured with a high resolution coaxial germanium detector which was set to cover the energy range up to $2 \mathrm{MeV} .{ }^{25}$ The germanium crystal provided a $20 \%$ efficiency at $1.33 \mathrm{MeV}$ with an energy resolution of $1.6 \mathrm{keV}$ (FWHM). The pulse height measurement (Fig. 7) showed that a series of $\gamma$ lines are present which can be ascribed to the radioactive decay chains of ${ }^{238} \mathrm{U}$ and ${ }^{235} \mathrm{U}$ isotopes of the analyser foil. Among the many peaks of Fig. 7 one can identify the line at $185.7 \mathrm{keV}$ as a $\gamma$ decay line of ${ }^{235} \mathrm{U}$ and the line at $92 \mathrm{keV}$ as $\mathrm{x}$-ray emission from thorium isotopes; the latter are produced from the decay chains of the two uranium isotopes.

The YAP background and signal features identified in the TOF spectrum can be studied by analyzing the $\gamma$ pulse height spectra associated to different time regions. Shown in Fig. 8 is the spectrum for the time region $>10000 \mu \mathrm{s}$. The spectrum is dominated by the radioactivity of the uranium analy-

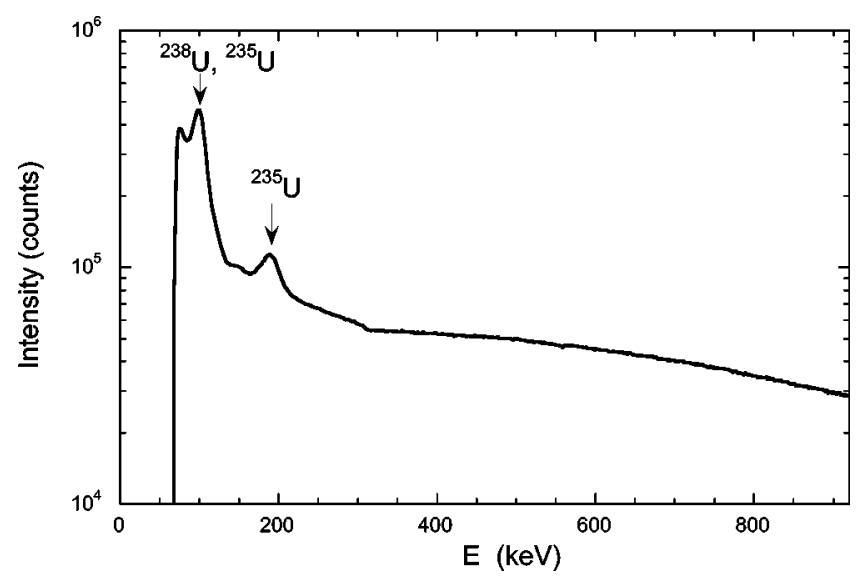

FIG. 8. Pulse height spectrum from the $\mathrm{Pb}$ sample obtained by projecting the biparametric data on the energy axis in the time interval $10000<t<20000 \mu \mathrm{s}$. 


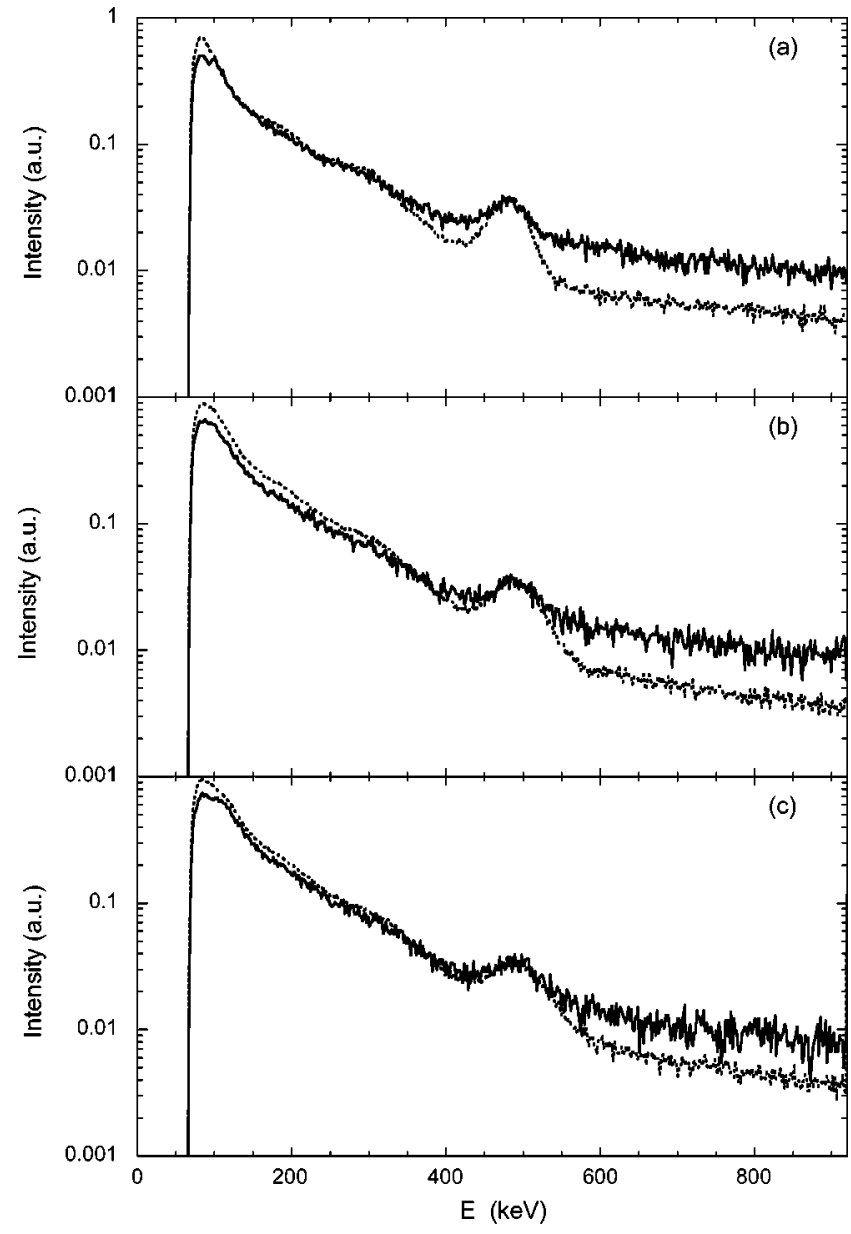

FIG. 9. Pulse height spectrum from the $\mathrm{Pb}$ sample obtained projecting the biparametric data on the energy axis for specified time intervals. In each panel a pair of normalized spectra are shown corresponding to a narrow time window around the neutron resonance (continuous line) and an offresonance region (dashed line). The chosen time intervals are (a) $309.0<t<313.0 \mu \mathrm{s}$ (resonance) and $272.0<t<299.0 \mu$ s (off-resonance); (b) $173.5<t<175.0 \mu \mathrm{s}$ (resonance) and $145.0<t<168.0 \mu \mathrm{s}$ (offresonance); (c) $130.25<t<131.25 \mu \mathrm{s}$ (resonance) and $115.0<t<128.0 \mu \mathrm{s}$ (off-resonance).

ser foil. A direct comparison with Fig. 7 shows that the two major peaks in the spectrum can be ascribed to the decay chains of ${ }^{238} \mathrm{U}$ and ${ }^{235} \mathrm{U}$. The YAP pulse height response to neutron absorption in the ${ }^{238} \mathrm{U}$ analyzer foil is shown in Figs. 9(a)-9(c) for the neutron resonances located at 6.67, 20.86, and $36.68 \mathrm{eV}$. The two spectra in each panel correspond to the resonance (continuous line) and off-resonance (dashed line) energy spectra. Each pair of $\gamma$ energy spectra was produced by selecting narrow time windows around each neutron resonance in the TOF spectrum of Fig. 5 and broad windows in the off-resonance regions, and by projecting the biparametric data along the energy axis. For instance, the pair of energy spectra of Fig. 9(a) were generated by selecting a $4 \mu \mathrm{s}$ wide time window centered around $t=309.5 \mu \mathrm{s}$, i.e., the $6.671 \mathrm{eV}$ resonance, and the region $272-299 \mu \mathrm{s}$, i.e., an off-resonance interval. In each panel the two spectra have been normalized to the height of the ${ }^{10} \mathrm{~B}$ peak, which provides a convenient normalization point. For $E_{\gamma}<400 \mathrm{keV}$ the spectra show essentially the same features. Earlier measurements performed with CZT detectors in the energy range
20-200 keV (Ref. 10) indicated that small differences exist between the resonance and off-resonance spectra but they cannot be distinguished by the YAP detector due to its poor energy resolution. The structure at $478 \mathrm{keV}$ has been previously identified as photons produced neutron absorption in ${ }^{10} \mathrm{~B}$. Above $500 \mathrm{keV}$ the on-resonance spectrum is systematically higher (about a factor two) than the off-resonance spectrum. These events are due to the Compton interactions of the radiative capture $\gamma$ rays of high energy (above $700 \mathrm{keV}$ ). The higher on-resonance spectrum level represents a clear signal signature which suggests that an improvement of the signal to background ratio of the TOF measurement can be obtained by increasing the $\gamma$ energy discrimination. This occurs at the cost of a reduced counting statistics. In order to investigate the best tradeoff between high signal-tobackground ratio and high counting statistics, a figure of merit $(F)$ can be defined. Here $F$ is defined as the reciprocal of the relative statistical error on the number of signal counts $(S)$ under the recoil peak, i.e.,

$$
F=\left(\frac{\sigma_{S}}{S}\right)^{-1}
$$

Indicating with $B$ the number of background counts under the peak, one can express the total number of counts as $\varepsilon$ $=S+B$ and the signal-to-background ratio as $q=S / B . F$ can thus be expressed merely as a function of the variables $\varepsilon$ and $q$ as follows. The relative statistical error on the number of signal counts under the recoil peak is

$$
\left(\frac{\sigma_{S}}{S}\right)=\frac{\sqrt{\sigma_{\varepsilon}^{2}+\sigma_{B}^{2}}}{S} .
$$

Assuming a normal distribution for $S$ and $B$ we have that $\sigma_{\varepsilon}^{2}=\varepsilon$ and $\sigma_{B}^{2}=B$ and Eq. (5) can be thus rewritten as

$$
\left(\frac{\sigma_{S}}{S}\right)=\frac{\sqrt{\varepsilon+B}}{\varepsilon-B}
$$

From the definition of $\varepsilon$ and $q$ it is trivial to see that $B$ can be expressed as

$$
B=\frac{\varepsilon}{1+q} \text {. }
$$

Now using Eq. (6) and Eq. (7) $F$ can be expressed as

$$
F=\frac{q}{\sqrt{(1+q)(2+q)}} \sqrt{\varepsilon} .
$$

The $\varepsilon$ and $q$ values of the recoil peaks in the TOF spectrum can be calculated and are used to determine $F$ via Eq. (8). Such data analysis procedure has been repeated for TOF spectra generated with different LLD values to obtain the three curves (i) $q$ versus LLD, (ii) $\varepsilon$ versus LLD, and (iii) $F$ versus LLD. These curves are shown in Figs. 10-12 for the neutron resonances up to $36.68 \mathrm{eV}$ and for 16 different LLD values ranging from 80 to $900 \mathrm{keV}$.

\section{DISCUSSION}

The YAP response to the signal and background sources has been characterized with the pulse height spectra associated to different time regions (Fig. 9). A comparison of the 


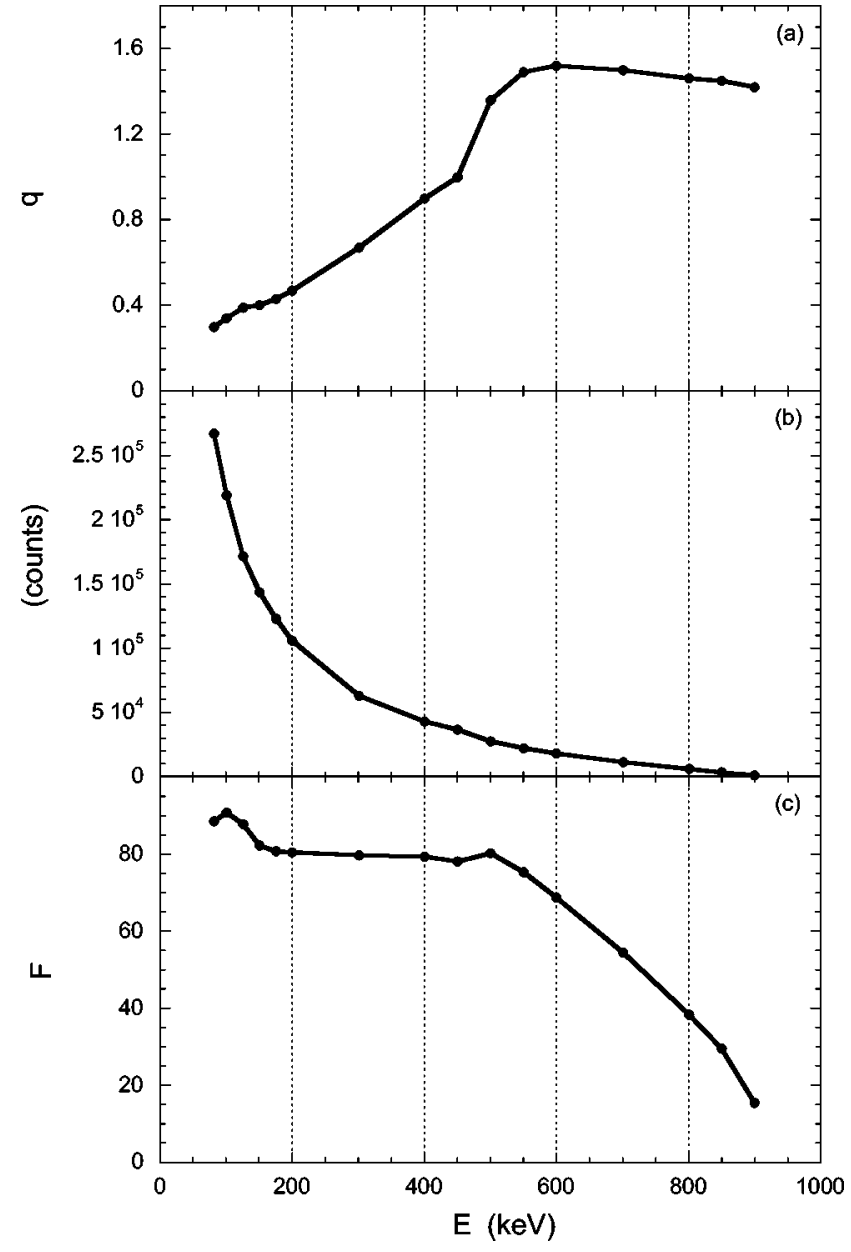

FIG. 10. Signal to background ratio $(q)$, counting efficiency $(\varepsilon)$, and figure of merit $(F)$ versus lower level discrimination (LLD) for the neutron resonance at $6.671 \mathrm{eV}$.

normalized pulse height spectra associated to resonance and off-resonance (background) regions seems to suggest that below $400 \mathrm{keV}$ the background is dominant over the signal and that the background and signal have similar spectral features. The signal is given by the prompt radiative capture $\gamma$ rays and $\mathrm{x}$ rays of the uranium analyser foil. The $\mathrm{x}$-ray photons deposit their full energy in the detector while the majority of the radiative capture $\gamma$ rays, due to the intrinsic low photopeak efficiency of the YAP crystal at energies above $300 \mathrm{keV}$, interact in the YAP crystal predominantly via Compton scattering. This results in signal events with a rather flat pulse height distribution which covers a broad energy range [Figs. 9(a)-9(c)]. The off-resonance spectra receive the contribution from several background components which are induced by the environmental background, time related to the neutron beam, and by the radioactivity of the analyzer foil. The former is made of $\mathrm{x}$-ray emission induced by absorption of nonresonant neutrons in ${ }^{238} \mathrm{U},{ }^{10}{ }^{10} \mathrm{~B}$ radiative capture photons and other unidentified $\gamma$ sources. The $\mathrm{x}$-ray emission component is the most intense one and, being present also for resonant neutron absorption, cannot be distinguished from the signal. ${ }^{10}$ The same happens for ${ }^{10} \mathrm{~B}$ radiative capture photons $\left(E_{\gamma}=478 \mathrm{keV}\right)$ which contribute to the pulse height spectra of Fig. 9 below the compton edge $\left(E_{\gamma}=311 \mathrm{keV}\right)$ and with the photopeak centered at $478 \mathrm{keV}$.

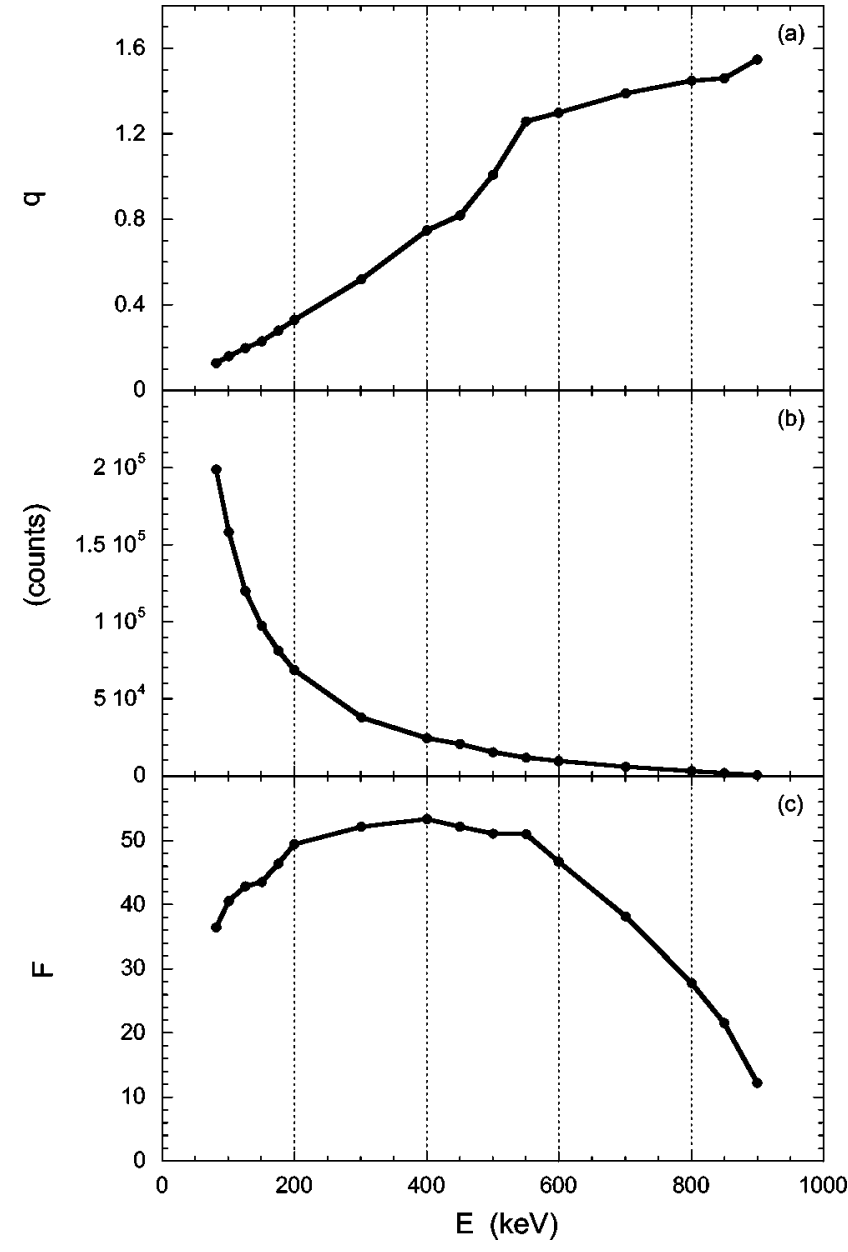

FIG. 11. Signal to background ratio $(q)$, counting efficiency $(\varepsilon)$, and figure of merit $(F)$ versus lower level discrimination (LLD) for the neutron resonance at $20.87 \mathrm{eV}$.

The second contribution to the background is represented by the radioactivity of the $\mathrm{U}$ analyzer foil and has therefore a flat time structure. Its pulse height spectrum (Fig. 7) measured with a Ge detector of high intrinsic photopeak efficiency $(>50 \%$ at $500 \mathrm{keV}$ ) shows that the majority (about $70 \%$ ) of the registered events are below $400 \mathrm{keV}$. The pulse height spectrum of YAP at long time of flights (Fig. 8) is indeed dominated by the analyzer foil radioactivity and has indicated that about $65 \%$ of the registered events are below $400 \mathrm{keV}$. Energy discrimination of the detected photons is an effective way to reduce the interference of the different background components. Shielding is also another possibility to reduce the background, except for the unavoidable uranium radioactivity, but is not practical due to space limitations; the effects of the shielding on scattered neutrons should also be considered. The potentiality of energy discrimination is discussed below.

Increasing the LLD threshold on the $\gamma$ pulse height permits us to discriminate the background at the price of losing some signal fraction. This has been quantified in Figs. 10-12 for the neutron resonances at 6.67, 20.86, and $36.68 \mathrm{eV}$. All three studied neutron resonances show similar features in the signal to background ratio (S/B) versus LLD curve [Figs. 10(a)-12(a)]. In the lower energy region $(80-400 \mathrm{keV})$ there is a linear increase in $S / B$ with increasing LLD values. Such 


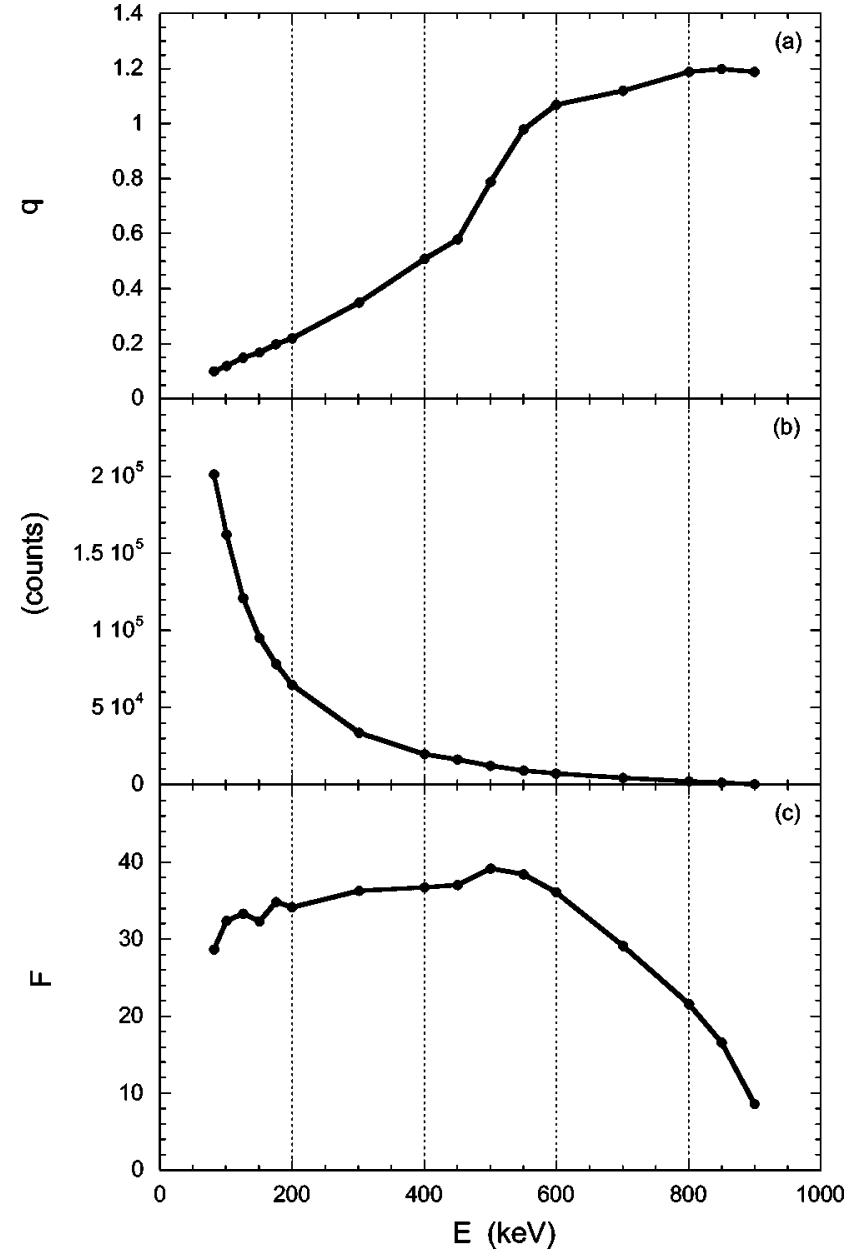

FIG. 12. Signal to background ratio $(q)$, counting efficiency $(\varepsilon)$, and figure of merit $(F)$ versus lower level discrimination (LLD) for the neutron resonance at $36.68 \mathrm{eV}$.

improvement can be attributed to the reduction of the background events induced by both uranium radioactivity and boron compton events. The central region $400-600 \mathrm{keV}$ is characterized by a steep rise in $S / B$ (about a factor 2), primarily due to the discrimination of the ${ }^{10} \mathrm{~B}$ photo-peak located at $478 \mathrm{keV}$. Above $600 \mathrm{keV}$ there is little improvement in $S / B$ and the curves seem to reach a plateau. In such an energy region all ${ }^{10} \mathrm{~B}$ background events and the majority of the uranium radioactivity have been discriminated, which makes the reduction that can be achieved in $\gamma$ background proportional to the reduction in the signal, as evident in the pulse height spectra of Fig. 9. Employing $\gamma$ energy discrimination there is, together with an improvement in $S / B$, a loss in counting statistics. This can be noticed from the plots of Figs. 10(b)-12(b) where the total numbers of counts under the recoil peaks show a rapid exponential decrease. The resulting statistical uncertainty in the signal as a function of LLD has been evaluated introducing the figure of merit $F$. The resonance at $6.67 \mathrm{eV}$ shows the best figure of merit, with a rather flat distribution around the average value of $F$ $=80$ up to $500 \mathrm{keV}$ [Fig. 10(c)]. Such value translates from the definition of $F$ [Eq. (4)] into about a $1 \%$ statistical error in the signal counts under the $6.67 \mathrm{eV}$ recoil peak. Increasing the LLD setting from 80 up to $500 \mathrm{keV}$ has a little effect on
$F$ since the improvement in $S / B$ is compensated from the decrease in counting statistics $\varepsilon$. The situation is partly different for the resonance at 20.86 and $36.68 \mathrm{eV}$, which are both characterized by a somewhat more peaked distribution of $F$, as shown in Figs. 11(c) and 12(c), respectively. The two plots seem to suggest that the optimum LLD setting is around $400-600 \mathrm{keV}$. In general the figure of merit for the three studied resonances indicates that the highest values of $F$ are reached for a quite broad range of LLD values. This means that the LLD setting is not critical and that the gain variation of the photomultiplier-tube, discussed in Sec. III A is indeed not affecting the measurement.

We observe that the curves found for $q, \varepsilon$, and $F$ are strictly valid only for one specific combination of sample $(\mathrm{Pb})$ and detector position. The detector angular position is less important in this case since $\mathrm{Pb}$ is an almost isotropic scatterer. A fraction of the $\gamma$ background is sample dependent which means that $S / B$ might change with different samples. The detector position plays also a role since by increasing the detector to sample distance $(d)$ the signal and the sampledependent background fraction drop as $1 / d^{2}$ while the uranium radioactivity background remains constant, which results in a reduction of $S / B$. The present discussion should therefore be considered as a qualitative indication of the performance of a YAP based detection system.

The above results indicate that for the higher energy resonances the optimum setting of the LLD is in the range $400-600 \mathrm{keV}$. This would not seem ideal from a $\gamma$ detection point of view since the YAP detector thickness is optimized for detecting photons below $200 \mathrm{keV}$. However other considerations need to be taken into account for the selection of the optimum crystal thickness. If, on one hand, a thicker crystal would provide higher $\gamma$ detection efficiency, on the other hand, it would also mean higher sensitivity to the $\gamma$ background. Moreover, neutron backscattering in the YAP crystal itself is a potential background source. Neutrons of slightly higher energy than the resonance energy are transmitted through the foil and can degrade their energy via backscattering in the YAP crystal toward the analyzer foil itself. Here they can be absorbed by the resonance thus creating an additional peak in the time-of-flight spectrum. The backscattering peak is too close to the main peak to be resolved and it would represent an undesired distortion of the peak shape. We remind here that the physics information that can be extracted from DINS measurement lies primarily in the shape of the recoil peak. Simulations will be carried on in order to investigate neutron backscattering and to confirm that the chosen YAP crystal thickness is indeed a good compromise between $\gamma$ detection efficiency and neutron backscattering. In principle a substantial improvement could occur by replacing the YAP scintillator with a higher $\mathrm{Z}$ (higher $\gamma$ efficiency) and lower neutron scattering cross section material.

\section{RD PROSPECTS AND APPLICATIONS}

This work describes a series of scattering experiments performed on the VESUVIO neutron spectrometer setup in the RD configuration. The prospects of the RD to be a suit- 
able configuration for neutron spectroscopy in the electron volt energy range are primarily based on the ability of the detector system to detect neutrons of final energies of tens of $\mathrm{eV}$ with good signal to background discrimination. The measurements performed with a single YAP scintillator and a natural uranium analyzer foil allowed us to detect scattered neutrons with final neutron energies up to $200 \mathrm{eV}$.

The response of a YAP scintillator to radiative capture $\gamma$ emission from a uranium analyzer foil was characterized with a biparametric data acquisition, which consisted of a simultaneous measurement of the neutron time of flight and $\gamma$ pulse height spectra. The analysis of the $\gamma$ pulse height spectra associated with resonant and non-resonant neutron absorption has allowed us to identify the different signal and background components. The signal is predominantly made up of compton events induced by radiative capture $\gamma$ rays which are not fully stopped in the detector. These signal events are superimposed on a background whose main sources have been identified as (i) $\mathrm{x}$ rays induced by absorption of nonresonant neutron in ${ }^{238} \mathrm{U}$, (ii) ${ }^{10} \mathrm{~B}$ radiative capture photons, and (iii) radioactivity of the $\mathrm{U}$ analyzer foil. The components (i) and (ii) are time related to the neutron beam while the component (iii) gives a flat contribution at all times. The quality of the TOF measurements depends on the relative intensity of the signal recoil peaks to the background, i.e., the signal to background ratio $S / B$. It has been shown that energy discrimination is an effective method to reduce the background interference. A significant improvement in $S / B$ has been achieved by using a lower level discrimination (LLD) of the $\gamma$ energies at about $600 \mathrm{keV}$. A study, based on a figure of merit $(F)$, of the achievable statistical uncertainty in the signal recoil peaks has shown that the best results are reached for a quite broad range of LLD values.

Two applications of the RD are discussed here. The first one is to access the region of high wave vector transfers $\left(q>100 \AA^{-1}\right)$ coupled to high energy transfers $(\omega>1 \mathrm{eV})$ in order to perform a deep inelastic neutron scattering (DINS) experiment. An example of a DINS experiment is represented by the measurement of a $\mathrm{H}_{2} \mathrm{O}$ sample at a scattering angle of about $30^{\circ} .{ }^{24}$ Neutrons scattered from the $\mathrm{H}_{2} \mathrm{O}$ sample were measured with a single YAP detector viewing a $30 \mu \mathrm{m}$ thick ${ }^{238} \mathrm{U}$ analyzer. In order to achieve a high counting statistics over a short time the LLD was set to a low photon energy value $(50 \mathrm{keV})$ at the price of increased background sensitivity. A typical time of flight spectrum recorded by YAP is shown in Fig. 13 for the case of $\mathrm{H}_{2} \mathrm{O}$ sample at $T=300 \mathrm{~K}$ and $p=1 \mathrm{bar}$ in an $\mathrm{Al}$ container. The peaks can be ascribed to scattering from the $\mathrm{H}$ and the $\mathrm{Al}$ atoms; they are separated because of their mass difference. We note that $\mathrm{H}$ and $\mathrm{Al}$ recoil peaks can be distinguished from the background up to final neutron energies of $66.0 \mathrm{eV}$. Final aim of the data analysis is the determination of the $\mathrm{H}$ mean kinetic energy. Data analysis has shown that the experimental response function and $\mathrm{H}$ mean kinetic energy are in agreement with previous experiments on this sample. ${ }^{24}$

A second application of the RD is the construction of the very low angle detector (VLAD) bank of the VESUVIO spectrometer which will cover the $1^{\circ}-5^{\circ}$ angular range at a

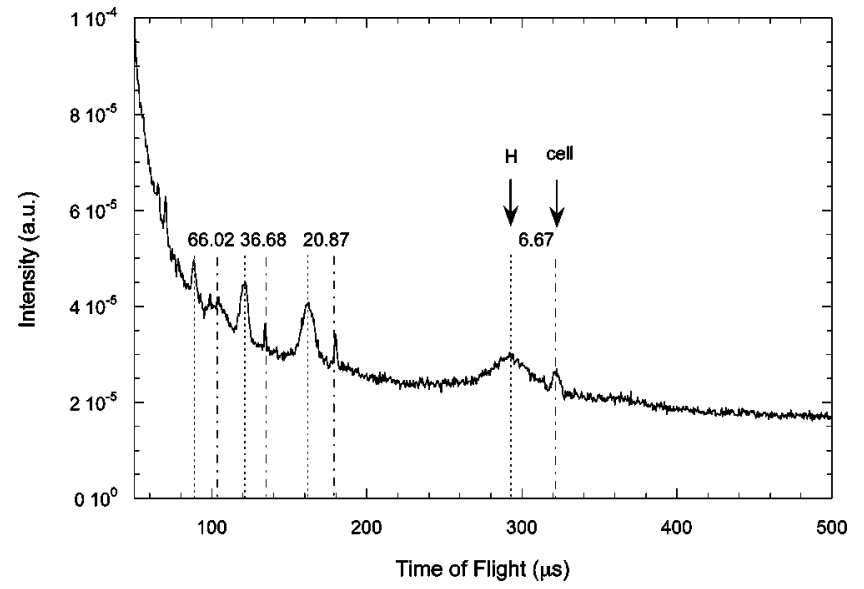

FIG. 13. Time of flight spectrum registered from a $\mathrm{H}_{2} \mathrm{O}$ sample in an $\mathrm{Al}$ cell in the time region 50-500 $\mu \mathrm{s}$. The dashed and dot-dashed lines indicate the $\mathrm{H}$ and cell recoil peaks, respectively, corresponding to the ${ }^{238} \mathrm{U}$ resonances at the energies (in electron volts) indicated by the labels.

distance of about $2 \mathrm{~m}$ from the scattering sample. VLAD will extend the kinematical region for neutron scattering to low wave vector transfer $\left(<10 \AA^{-1}\right)$ while keeping energy transfer $>1 \mathrm{eV}$. Accessing such kinematical region will allow experimental studies in condensed matter systems. The first step of the upgrade was the installation of a vacuum tank, specifically designed in order not to intercept neutrons scattered at very low angles. A detector bank prototype was built consisting of four RD detectors, each one made of two YAP crystals of trapezoidal shape enclosed in an aluminum case which acts as a light guide. A $25 \mu \mathrm{m}{ }^{238} \mathrm{U}$ analyzer foil was attached to each RD unit. Measurements were taken from an ice sample at $270 \mathrm{~K}$ at scattering angles varying between $2^{\circ}$ and $5^{\circ}$. The four VLAD detectors covered the energy transfer range of $0.2-20 \mathrm{eV}$ and the wave vector transfer range of 3-10 $\AA^{-1}$. A preliminary analysis of the resonance at $6.67 \mathrm{eV}$ has indicated that one can derive quantitative values for the dynamical structure factor $S(q, \omega)$ and the density of states $g(\omega)$, of hydrogen in the sample using the same method for data analysis of previous experiments performed on a different spectrometer. ${ }^{26}$ These measurements are the first of this kind on an inverse geometry instrument.

The present results indicate YAP scintillator as the ideal RD candidate for studies of microscopic dynamics of matter via epithermal neutron scattering. YAP scintillators appear as most suitable RD for inelastic neutron scattering at both intermediate and very low scattering angles. Further development can be done to improve the detection efficiency and the neutron energy resolution. Other issues being addressed in the near future are cross talk between nearby detectors and multiple neutron scattering. These are relevant issues in view of the planned construction of a position sensitive RD array.

\section{ACKNOWLEDGMENTS}

This work was performed with financial support by the European Community-Access to Research Infrastructure action of the Improving Human Potential Program (EU Project HPRI-CT-2001-50020). We acknowledge Consiglio Nazion- 
ale delle Ricerche (CNR)-Italy for financial support for the experiments performed at the ISIS pulsed neutron source.

${ }^{1}$ S. W. Lovesey, Theory of Neutron Scattering from Condensed Matter, (Oxford University Press, London, 1987).

${ }^{2}$ G. Watson, J. Phys.: Condens. Matter 8, 5955 (1996).

${ }^{3}$ Proceedings of the 1984 Workshop on High-Energy Excitations in Condensed Matter, Los Alamos, New Mexico, February 1984.

${ }^{4}$ A. Pietropaolo, R. Senesi, M. Tardocchi, C. Andreani, and G. Gorini, Physica B 350, e857 (2004).

${ }^{5}$ C. Andreani, A. Pietropaolo, R. Senesi, G. Gorini, M. Tardocchi, A. Bracco, N. Rhodes, and E. Schoneweld, Nucl. Instrum. Methods Phys. Res. A 481, 509 (2002).

${ }^{6}$ C. Andreani, A. D’Angelo, G. Gorini, S. Imberti, A. Pietropaolo, N. Rhodes, E. Schooneveld, R. Senesi, and M. Tardocchi, Appl. Phys. A: Mater. Sci. Process. 78, 903 (2004).

${ }^{7}$ D. R. Allen, E. W. J. Mitchell, and R. N. Sinclair, J. Phys. E 13, 639 (1980).

${ }^{8}$ J. M. Carpenter, N. Watanabe, S. Ikeda, Y. Masuda, and S. Sato, Physica B\&C 120, 126 (1983).

${ }^{9}$ M. Tardocchi, C. Arnaboldi, G. Gorini, S. Imberti, G. Pessina, E. Previtali, C. Andreani, A. Pietropaolo, and R. Senesi, Physica B 350/1-3S, E853 (2004).

${ }^{10}$ M. Tardocchi, A. Pietropaolo, C. Andreani, A. Bracco, A. D’Angelo, G. Gorini, S. Imberti, R. Senesi, N. Rhodes, and E. S. Schooneveld, Nucl. Instrum. Methods Phys. Res. A 526/3, 279 (2004).

${ }^{11}$ A. A. Fyodorov, A. L. Kholmetskii, M. V. Korzhik, A. R. Lopatik, M. Mashlan, and O. V. Misevich, Nucl. Instrum. Methods Phys. Res. B 88, 462 (1994); A. L. Kholmetskii, M. Mashlan, O. V. Misevich, V. A. Chudakov, A. R. Lopatik, and D. Zak, Nucl. Instrum. Methods Phys. Res. B 124, 143 (1997)
${ }^{12}$ K. Hirota, H. Toyokawa, M. Suzuki, T. Kudo, M. Nomachi, Y. Sugaya, M. Yosoic, A. Gorind, I. Manuilovd, A. Riazantsevd, and K. Kuroda Nucl. Instrum. Methods Phys. Res. A 510, 83 (2003).

${ }^{13}$ F. Vittori, F. de Notaristefani, and T. Malatesta, Nucl. Phys. B 78, 616 (1999).

${ }^{14}$ R. Senesi, C. Andreani, Z. Bowden, D. Colognesi, E. Degiorgi, A. L. Fielding, J. Mayers, M. Nardone, J. Norris, M. Praitano, N. J. Rhodes, W. G. Stirling, J. Tomkinson, and C. Uden, Physica B 276-278, 200 (2000).

${ }^{15}$ A. L. Fielding and J. Mayers, Nucl. Instrum. Methods Phys. Res. A 480, 680 (2002).

${ }^{16}$ Bicron product Catalogue, http://www. bicron. com

${ }^{17}$ Data compiled by C. M. Rozsa, Bicron Brochure, "Efficiency for selected scintillators," http://www. bicron. com

${ }^{18}$ A. Pietropaolo, C. Andreani, A. D’Angelo, G. Gorini, S. Imberti, N. Rhodes, E. Schooneveld, R. Senesi, and M. Tardocchi, Appl. Phys. A: Mater. Sci. Process. 74, S189 (2002).

${ }^{19}$ S. F. Mughabghab, Neutron Cross Sections, Vol. 1, Part B (Academic, New York, 1984).

${ }^{20}$ National Nuclear Data Center, Brookhaven National Laboratory, http:// www.nndc.bnl.gov and Thermal Neutron Capture Home Page, Ernest O. Lawrence Berkeley National Laboratory, http://ie.lbl.gov/ng.html

${ }^{21}$ M. Tardocchi, S. Conroy, G. Ericsson, J. Frenje, J. Källne, and E. Traneus, Nucl. Instrum. Methods Phys. Res. A 485, 624 (2002).

${ }^{22}$ A. Michalowicz, Kinematics of Nuclear Reactions (Iliffe Bools Ltd., London, 1967).

${ }^{23}$ W. E. Lamb, Phys. Rev. 55, 190 (1939).

${ }^{24}$ A. Pietropaolo et al. (unpulblished).

${ }^{25}$ M. Tardocchi, Background in the VESUVIO Experimental Hall: First Measurements with a Germanium Detector, November 2002, Internal Report of Physics Department "G. Occhialini," Milano-Bicocca University, Milan, Italy.

${ }^{26}$ C. Andreani et al., Appl. Phys. Lett. (accepted). 